\title{
The Hochschild Cohomology Group of a Class of Directed Tree-Path Algebras

\author{
Yanfeng $\mathrm{Li}^{1}$, Haicheng Liu ${ }^{1 *}$, Wenyan Kang ${ }^{2}$, Guiying Zhu ${ }^{1}$
}

${ }^{1}$ College of Science, Heilongjiang Bayi Agricultural University, Daqing 163319, Heilongjiang, P. R. China

${ }^{2}$ College of Science, Qiqihar University, Qiqihar 161006, Heilongjiang, P. R. China

DOI: $10.36347 /$ sjet.2020.v08i06.002

| Received: 18.06.2020 | Accepted: 25.06.2020 | Published: 28.06.2020

*Corresponding author: Haicheng Liu

Abstract

Review Article

The Hochschild cohomology of associative algebra is closely related to its algebraic structure. According to the characteristics of double modules in directed tree-path algebra and Hochschild's theory, the Hochschild cohomology groups of some finite dimensional algebras have been studied deeply. In this paper, we calculate the Hochschild cohomology groups of a directed tree-path algebra with and without branches.

Keywords: Hochschild cohomology group; Path algebras; Directed graph.

Copyright @ 2020: This is an open-access article distributed under the terms of the Creative Commons Attribution license which permits unrestricted use, distribution, and reproduction in any medium for non-commercial use (NonCommercial, or CC-BY-NC) provided the original author and source are credited.

\section{INTRODUCTION}

The homology group theory of associative algebra is very rich [1, 2], before the homology group theory of associative algebra was defined, people only studied some special theories of derivatives and extensions. Since Hochschild proposed the Hochschild group theory of finite dimensional associative algebra in 1945, it becomes a meaningful subject to use the Hochschild group theory to study finite dimensional algebra [3-5]. The low order Hochschild cohomology groups are closely related to the algebraic structure. Therefore, the calculation of Hochschild cohomology groups of various algebras is of great significance in algebraic representation theory. This paper mainly studies a class of Hochschild cohomology groups of directed tree-path algebra.

\section{Preparative knowledge}

Definition 1 [6] Assume $A_{i}, A, B, C$ are $R$ - modules, $i \in I$, then the following $R$ - modules are isomorphic:

$$
\begin{aligned}
& A \otimes_{R} B \cong B \otimes_{R} A \\
& R \otimes_{R} A \cong A \text {. } \\
& \left(\underset{i \in I}{\oplus A_{i}}\right) \otimes_{R} B \cong \oplus\left(A_{i} \otimes_{R} B\right) \\
& \left(A \otimes_{R} B\right) \otimes_{R} C \cong A \otimes_{R}\left(B \otimes_{R} C\right)
\end{aligned}
$$

Definition 2 [7] Assume $k$ is a domain, $Q$ is a directed graph, $A=k Q$ is a $k$-vector space based on the path of $Q$. For $p=\alpha_{1} \cdots \alpha_{m}$ and $q=\beta_{1} \cdots \beta_{n}$, define multiplication:

$$
p q=\left\{\begin{array}{cc}
\alpha_{1} \cdots \alpha_{m} \beta_{1} \cdots \beta_{n}, & t(p)=s(q) \\
0, & t(p) \neq s(q)
\end{array}\right.
$$

In this case, $A=k Q$ is an $k$-algebra, we called it path algebra of $\mathrm{Q}$, path algebra for short. 
Definition 3 [8] Assume $A$ is a finite dimensional $k$-algebra, $M$ is a finite dimensional $A$ - $A$ - bimodule. $C=\left(C^{i}, d^{i}\right)(i \in Z)$

$C=\cdots \rightarrow C^{-1} \stackrel{d^{-1}}{\longrightarrow} C^{0} \stackrel{d^{0}}{\longrightarrow} C^{1} \rightarrow \cdots \rightarrow C^{i} \stackrel{d^{i}}{\longrightarrow} C^{i+1} \rightarrow \cdots$

Is called Hochschild complex, where $C^{i}=0, d^{i}=0, \forall i<0, C^{0}=M, C^{i}=\operatorname{Hom}_{k}\left(A^{\otimes i}, M\right), \forall i>0$.

$A^{\otimes i}$ Represents that A makes i-th tensor product with itself on the field k.

$$
\begin{aligned}
& d^{0} m(a)=a m-m a, \forall m \in M, a \in A \\
& \left(d^{i} f\right)\left(a_{1} \otimes \cdots \otimes a_{i+1}\right)=a_{1} f\left(a_{2} \otimes \cdots \otimes a_{i+1}\right) \\
& +\sum_{j=1}^{i}(-1)^{j} f\left(a_{1} \otimes \cdots \otimes a_{j} a_{j+1} \otimes \cdots \otimes a_{i+1}\right)+(-1)^{i+1} f\left(a_{1} \otimes \cdots \otimes a_{i}\right) a_{i+1}
\end{aligned}
$$

With $f \in \operatorname{Hom}_{k}\left(A^{\otimes i}, M\right)$. Denote $H^{i}(A, M)=H^{i}(C)=\operatorname{Kerd}^{i} / \operatorname{Im} d^{i-1}, \forall i \in Z$, it is called the i-th Hochschild cohomology group of the coefficient of $A$ in $M$.

In particular, when we take $M=A, H^{i}(A)=H^{i}(A, A)$ is called the i-th Hochschild cohomology group of algebra A.

\section{The Hochschild Cohomology Group of a Directed Tree-path Algebras without branches}

Proposition 2.1 Assume $D$ is a finite dimensional directed tree graph without branches, $D_{0}=\left\{e_{0}, e_{1}\right\}$ is a Vertex set, $D_{1}=\left\{a_{1}\right\}$ is a set of directed edges with length $1, C$ is a Path algebra of $D$ over $K$, then $H^{0}(C) \cong K \oplus K$, $H^{1}(C)=0$.

Proof According to the known conditions, the directed tree graph is $e_{0} \stackrel{a_{1}}{\longrightarrow} e_{1}$.

For $\forall x \in \operatorname{Kerd}_{1}, d_{1}(x)=0$. Assume $x=K_{1} e_{0}+K_{2} e_{1}+K_{3} a_{1}$, we have $d_{1}\left(K_{1} e_{0}+K_{2} e_{1}+K_{3} a_{1}\right)=K_{1} \Delta\left(e_{0}\right)+K_{2} \Delta\left(e_{1}\right)+K_{3} \Delta\left(a_{1}\right)=K_{3}\left(e_{0} \otimes e_{1}\right)=0$,

Therefore $K_{3}=0$, so $x=K_{1} e_{0}+K_{2} e_{1}$, Hence $H^{0}(C) \cong K \oplus K$.

In the following, we calculate $H^{1}(C)$.

For $\forall x \in \operatorname{Ker}\left(d_{2}\right), d_{2}(x)=0$. Let $x=K_{1} e_{0} \otimes a_{1}+K_{2} e_{0} \otimes e_{1}$, according to $\Delta\left(a_{i}\right)=e_{i-1} \otimes e_{i}, \Delta e_{i}=0$, we can derive that

$$
\begin{aligned}
d_{2}(x) & =(\Delta \otimes I-I \otimes \Delta) x \\
& =K_{1} \Delta\left(e_{0}\right) \otimes a_{1}+K_{2} \Delta\left(e_{0}\right) \otimes e_{1}+K_{3} \Delta\left(a_{1}\right) \otimes e_{1} \\
& -K_{1} e_{1} \otimes \Delta\left(a_{1}\right)-K_{2} e_{0} \otimes \Delta\left(e_{1}\right)-K_{3} a_{1} \otimes \Delta\left(e_{1}\right) \\
& =K_{3} e_{0} \otimes e_{1} \otimes e_{1}-K_{1} e_{1} \otimes e_{0} \otimes e_{1}=0
\end{aligned}
$$

Therefore $K_{1}=K_{3}=0$, so $x=K_{2}\left(e_{0} \otimes e_{1}\right)=K_{2} \Delta\left(a_{2}\right) \in \operatorname{Im} d_{1}$, Hence $\operatorname{Kerd} d_{2} \subset \operatorname{Im} d_{1}$. And because $d_{2} d_{1}=0$, we can obtain $\operatorname{Im} d_{1} \subset \operatorname{Kerd}{ }_{2}$, so $\operatorname{Kerd} d_{2}=\operatorname{Im} d_{1}$, hence $H^{1}(C)=0$.

Proposition 2.2 Assume $D$ is a finite dimensional directed tree graph without branches, $D_{0}=\left\{e_{0}, e_{1}, e_{2}\right\}$ is a Vertex set, $D_{1}=\left\{a_{1}, a_{2}\right\}$ is a set of directed edges with length $1, C$ is a Path algebra of $D$ over $K$, then $H^{0}(C) \cong K \oplus K \oplus K, H^{1}(C)=0$.

Proof According to the known conditions, the directed tree graph is $e_{0} \stackrel{a_{1}}{\longrightarrow} e_{1} \stackrel{a_{2}}{\longrightarrow} e_{2}$. 
For $\forall x \in \operatorname{Kerd}_{1}, d_{1}(x)=0$. Let $x=K_{1} e_{0}+K_{2} e_{1}+K_{3} e_{2}+K_{4} a_{1}+K_{5} a_{2}+K_{6} a_{1} a_{2}$, we have

$$
\begin{aligned}
& d_{1}(x)=\Delta(x)=K_{1} \Delta\left(e_{0}\right)+K_{2} \Delta\left(e_{1}\right)+K_{3} \Delta\left(e_{2}\right)+K_{4} \Delta\left(a_{1}\right)+K_{5} \Delta\left(a_{2}\right)+K_{6} \Delta\left(a_{1} a_{2}\right) \\
& =K_{4}\left(e_{0} \otimes e_{1}\right)+K_{5}\left(e_{1} \otimes e_{2}\right)+K_{6}\left(a_{1} \otimes e_{2}+e_{0} \otimes a_{2}\right)=0,
\end{aligned}
$$

Then $K_{4}=K_{5}=K_{6}=0$, therefore $x=K_{1} e_{0}+K_{2} e_{1}+K_{3} e_{2}$, hence $H^{0}(C) \cong K \oplus K \oplus K$.

In the following, we calculate $H^{1}(C)$.

$$
\text { For } \begin{aligned}
\forall x & \in \operatorname{Ker}\left(d_{2}\right), \text { let } \\
x & =K_{1}\left(e_{0} \otimes a_{1}\right)+K_{2}\left(e_{0} \otimes e_{1}\right)+K_{3}\left(e_{0} \otimes a_{2}\right)+K_{4}\left(e_{0} \otimes e_{2}\right)+K_{5}\left(a_{1} \otimes e_{1}\right) \\
& +K_{6}\left(a_{1} \otimes a_{2}\right)+K_{7}\left(a_{1} \otimes e_{2}\right)+K_{8}\left(e_{1} \otimes a_{2}\right)+K_{9}\left(e_{1} \otimes e_{2}\right)+K_{10}\left(a_{2} \otimes e_{2}\right),
\end{aligned}
$$

According to $d_{2}(x)=(\Delta \otimes I-I \otimes \Delta) x=0$, we obtain

$K_{1}=K_{4}=K_{5}=K_{6}=K_{8}=K_{10}=0, K_{3}=K_{7}, K_{2}, K_{9}$ are free variables.

Then we have

$$
\begin{aligned}
x & =K_{3}\left(e_{0} \otimes a_{2}+a_{1} \otimes e_{2}\right)+K_{2}\left(e_{0} \otimes e_{1}\right)+K_{9}\left(e_{1} \otimes e_{2}\right) \\
& =K_{3} \Delta\left(a_{1} a_{2}\right)+K_{2} \Delta\left(a_{1}\right)+K_{9} \Delta\left(a_{2}\right) \in \operatorname{Im} d_{1}
\end{aligned}
$$

Therefor $\operatorname{Kerd}_{2} \subset \operatorname{Im} d_{1}$. And because $d_{2} d_{1}=0$, thus $\operatorname{Im} d_{1} \subset \operatorname{Ker} d_{2}$, we derive that $\operatorname{Kerd}{ }_{2}=\operatorname{Im} d_{1}$, hence $H^{1}(C)=0$.

Proposition 2.3 Assume $D$ is a finite dimensional directed tree graph without branches, $D_{0}=\left\{e_{0}, e_{1}, e_{2}, \cdots, e_{n}\right\}$ is a Vertex set, $D=\left\{a_{1}, a_{2}, \cdots, a_{n}\right\}$ is a set of directed edges with length $1, C$ is a Path algebra of $D$ over $K$,then

$$
H^{0}(C) \cong \overbrace{K \oplus K \oplus \cdots \oplus K}^{n+1 \text { 个 }}, H^{1}(C)=0
$$

Proof By $e_{0} \stackrel{a_{1}}{\longrightarrow} e_{1} \stackrel{a_{2}}{\longrightarrow} e_{2} \stackrel{a_{3}}{\longrightarrow} \cdots \longrightarrow e_{n-1} \stackrel{a_{n}}{\longrightarrow} e_{n}$, we structure complex

$C \stackrel{\Delta=d_{1}}{\longrightarrow} C \otimes C \stackrel{d_{2}}{\longrightarrow} C \otimes C \otimes C \stackrel{d_{3}}{\longrightarrow} \cdots \stackrel{d_{n}}{\longrightarrow} C^{\otimes n+1}$.

For $\forall x \in \operatorname{Kerd}_{1}$, let

$x=K_{1} e_{0}+K_{2} e_{1}+\cdots+K_{n+1} e_{n}+l_{1} a_{1}+\cdots+l_{n} a_{n}+\sum_{i \in N^{+}} t_{i} a_{1} a_{2} \cdots a_{i}$,

By $d(x)=\Delta(x)=0$, we obtain $l_{i}=0(i=1,2, \cdots, n), t_{i}=0\left(i \in N^{+}\right)$, thus

$x=K_{1} e_{0}+K_{2} e_{1}+\cdots+K_{n+1} e_{n}$

So $H^{0}(C) \cong \overbrace{K \oplus K \oplus \cdots \oplus K}^{n+1 \uparrow}$.

In the following, we prove $H^{1}(C)=0$.

For $\forall x \in \operatorname{Ker}\left(d_{2}\right)$, we have $d_{2}(x)=0$. Because

$$
\begin{aligned}
d_{2}(x) & =(\Delta \otimes I-I \otimes \Delta) x \\
& =(\Delta \otimes I-I \otimes \Delta)\left(\sum K_{j_{1}}\left(e_{i_{1}} \otimes a_{t_{1}}\right)+\sum K_{j_{2}}\left(a_{i_{2}} \otimes e_{t_{2}}\right)+\sum K_{j_{3}}\left(e_{i_{3}} \otimes e_{t_{3}}\right)\right)=0
\end{aligned}
$$

Therefore

$$
\begin{aligned}
x & =\sum K_{j_{2}}\left(\Delta\left(a_{i_{2}}\right) \otimes e_{t_{2}}\right)-\sum K_{j_{1}}\left(e_{i_{1}} \otimes \Delta\left(a_{t_{1}}\right)\right) \\
& =\sum K_{t} \Delta\left(a_{1} a_{2}+a_{1} a_{3}+\cdots+a_{1} a_{n}+a_{1} a_{2} a_{3}+\cdots+a_{1} a_{2} a_{3} \cdots a_{n}\right) \in \operatorname{Im} d_{1}
\end{aligned}
$$

We obtain $\operatorname{Kerd}_{2} \subset \operatorname{Im} d_{1}$, and because $d_{2} d_{1}=0$, so $\operatorname{Im} d_{1} \subset \operatorname{Kerd}_{2}$, hence $H^{1}(C)=0$. 


\section{The Hochschild Cohomology Group of a Directed Tree-path Algebras with branches}

Proposition 3.1 Assume $D$ is a finite dimensional directed tree graph with branches, $D_{0}=\left\{e_{0}, e_{1}, e_{2}, e_{3}\right\}$ is a Vertex set, $D_{1}=\left\{a_{1}, a_{2}, a_{3}\right\}$ is a set of directed edges with length $1, C$ is a Path algebra of $D$ over $K$, then $H^{0}(C) \cong K \oplus K \oplus K, H^{1}(C)=0$.

Proof According to the known conditions, the directed tree graph is

Let $x \in \operatorname{Ker}\left(d_{1}\right)$, then $d_{1}(x)=0$. Assume

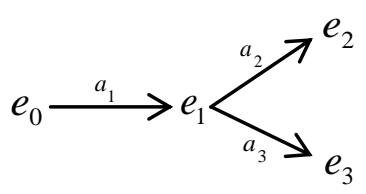
$x=K_{1} e_{0}+K_{2} e_{1}+K_{3} e_{2}+K_{4} e_{3}+K_{5} a_{1}+K_{6} a_{2}+K_{7} a_{3}+K_{8} a_{1} a_{2}+K_{9} a_{1} a_{3}$

According to

$d_{1}(x)=\Delta(x)=K_{5} \Delta\left(a_{1}\right)+K_{6} \Delta\left(a_{2}\right)+K_{7} \Delta\left(a_{3}\right)+K_{8} \Delta\left(a_{1} a_{2}\right)+K_{9} \Delta\left(a_{1} a_{3}\right)=0$

We obtain $K_{5}=K_{6}=K_{7}=K_{8}=K_{9}=0$, so $x=K_{1} e_{0}+K_{2} e_{1}+K_{3} e_{2}+K_{4} e_{3}$, hence we derive that $H^{0}(C) \cong K \oplus K \oplus K$.

In the following, we calculate $H^{1}(C)$.

For $\forall x \in \operatorname{Ker}\left(d_{2}\right)$, we have $d_{2}(x)=0$.

$$
\begin{aligned}
x & =K_{1}\left(e_{0} \otimes a_{1}\right)+K_{2}\left(e_{0} \otimes e_{1}\right)+K_{3}\left(e_{0} \otimes a_{2}\right)+K_{4}\left(e_{0} \otimes e_{2}\right)+K_{5}\left(a_{1} \otimes e_{1}\right) \\
& +K_{6}\left(a_{1} \otimes a_{2}\right)+K_{7}\left(a_{1} \otimes e_{2}\right)+K_{8}\left(e_{1} \otimes a_{2}\right)+K_{9}\left(e_{1} \otimes e_{2}\right)+K_{10}\left(a_{2} \otimes e_{2}\right) \\
& +K_{11}\left(e_{0} \otimes a_{3}\right)+K_{12}\left(e_{0} \otimes e_{3}\right)+K_{13}\left(a_{1} \otimes e_{1}\right)+K_{14}\left(a_{1} \otimes a_{3}\right)+K_{15}\left(a_{1} \otimes e_{3}\right) \\
& +K_{16}\left(e_{1} \otimes a_{3}\right)+K_{17}\left(e_{1} \otimes e_{3}\right)+K_{18}\left(a_{3} \otimes a_{3}\right) \\
& +K_{6}\left(a_{1} \otimes a_{2}\right)+K_{7}\left(a_{1} \otimes e_{2}\right)+K_{8}\left(e_{1} \otimes a_{2}\right)+K_{9}\left(e_{1} \otimes e_{2}\right)+K_{10}\left(a_{2} \otimes e_{2}\right)
\end{aligned}
$$

By $d_{2}(x)=(\Delta \otimes I-I \otimes \Delta) x=0$, we obtain $K_{11}=K_{15}, K_{3}=K_{7}, K_{2}, K_{9}, K_{17}$ are free variables, the others are both zero. Then

$$
\begin{aligned}
x & =K_{2}\left(e_{0} \otimes e_{1}\right)+K_{3}\left(e_{0} \otimes a_{2}+a_{1} \otimes e_{2}\right)+K_{9}\left(e_{1} \otimes e_{2}\right) \\
& +K_{11}\left(e_{0} \otimes a_{3}+a_{1} \otimes e_{3}\right)+K_{17}\left(e_{1} \otimes e_{3}\right)
\end{aligned}
$$

Thus we obtain $\operatorname{Ker} d_{2} \subset \operatorname{Im} d_{1}$, and because $d_{2} d_{1}=0$, so $\operatorname{Im} d_{1} \subset \operatorname{Ker} d_{2}$, hence $H^{1}(C)=0$.

Proposition 3.2 Assume $D$ is a finite dimensional directed tree graph with branches, $D_{0}=\left\{e_{0}, e_{1}, \cdots, e_{n}\right\}$ is a Vertex set, $D_{1}=\left\{a_{1}, a_{2}, \cdots, a_{n}\right\}$ is a set of directed edges with length $1, C$ is a Path algebra of $D$ over $K$,then

$$
H^{0}(C) \cong \overbrace{K \oplus K \oplus \cdots \oplus K}^{n+1 \uparrow}, H^{1}(C)=0 .
$$

Proof Firstly we calculate $H^{0}(C)$.

For $\forall x \in \operatorname{Kerd}_{1}$, let $x=K_{1} e_{0}+K_{2} e_{1}+\cdots+K_{n+1} e_{n}+\sum K_{l} a_{i 1} a_{i 2} \cdots a_{i n}$, by $\Delta(x)=0$, we obtain $K_{l}=0$.

Thus

$$
\begin{gathered}
x=K_{1} e_{0}+K_{2} e_{1}+\cdots+K_{n+1} e_{n} \\
\text { So } H^{0}(C) \cong \overbrace{K \oplus K \oplus \cdots \oplus K}^{n+1 \text { 个 }} .
\end{gathered}
$$

In the following, we calculate $H^{1}(C)$.

For $\forall x \in \operatorname{Ker}\left(d_{2}\right)$, we have $d_{2}(x)=0$. let

$$
x=\sum K_{i_{1}}\left(e_{j_{1}} \otimes a_{t_{1}}\right)+\sum K_{i_{2}}\left(a_{j_{2}} \otimes e_{t_{2}}\right)+\sum K_{i_{3}}\left(e_{j_{3}} \otimes e_{t_{3}}\right)
$$


By computing

$$
\begin{aligned}
d_{2}(x) & =(\Delta \otimes I-I \otimes \Delta) x \\
& =\sum K_{i_{2}}\left(\Delta\left(a_{j_{2}}\right) \otimes e_{t_{2}}\right)-\sum K_{i_{1}}\left(e_{j_{1}} \otimes \Delta\left(a_{t_{1}}\right)\right)=0
\end{aligned}
$$

We obtain $x=\sum L \Delta\left(a_{1} a_{2} a_{3} \cdots a_{n}\right) \subset \operatorname{Im} d_{1}$, therefor $\operatorname{Ker} d_{2} \subset \operatorname{Im} d_{1}$. And because $d_{2} d_{1}=0$, we have $\operatorname{Im} d_{1} \subset \operatorname{Kerd}_{2}$, hence $H^{1}(C)=0$.

\section{CONCLUSIONS}

In this paper, the Hochschild cohomology groups of a directed tree-path algebra with and without branches are calculated separately. We conclude that the zero-order Hochschild Homology results of directed tree-path algebra are not related to branches, but are related to the vertex set $D_{0}$ of the directed graph. Simultaneously we derive that the First Cohomology Group is trivial.

\section{FUNDING}

This research is supported by Heilongjiang Bayi Agricultural University Scientific Research Foundation for PhD (XDB-2016-25).

\section{REFERANCES}

1. Hochschild G. On the Cohomology groups of an associative algebra. Ann of Math. 1945, 46:58-67.

2. Hochschild G. On the Cohomology theory for an associative algebra. Ann of Math. 1946,47(2):568-579.

3. Cibis C. Hochschild Homology of an algebra whose quiver has no orienteal Cycles. LNM. 1986, 11(7):55-59.

4. Liu SX, Zhang P. Hochschild Homology of finite-dimensional Algebras.J.of pure and App algebra, 1993.

5. Liu SX, Zhang P. Hochschild Homology of finite-dimensional radical homogeneous algebras. Preprint, 1993.

6. Zhou B. Homology algebra. Higher Education Press, 2008.

7. Assen I, Simson D, Skowronski A. Elements of the Representation Theory of Associative Algebras. Vol.1. Techniques of representation theory. London Mathematical Society Student Texts 65. Cambridge: Cambridge University Press, 2006.

8. Carten H, Eilenberg S. Homological algebra. Princeton University Press, 1956. 